\title{
Multipoint Channel Charting for Wireless Networks
}

\section{Conference Paper}

Author(s):

Deng, Junquan; Medjkouh, Saïd; Malm, Nicolas; Tirkkonen, Olav; Studer, Christoph (D)

Publication date:

2018-10

Permanent link:

https://doi.org/10.3929/ethz-b-000461322

Rights / license:

In Copyright - Non-Commercial Use Permitted

Originally published in:

https://doi.org/10.1109/ACSSC.2018.8645281 


\title{
Multipoint Channel Charting for Wireless Networks
}

\author{
Junquan Deng*, Saïd Medjkouh ${ }^{\dagger}$, Nicolas Malm*, Olav Tirkkonen*, Christoph Studer ${ }^{\dagger}$ \\ *Department of Communications and Networking, Aalto University, Finland \\ ${ }^{\dagger}$ School of Electrical and Computer Engineering, Cornell University, Ithaca, NY, USA
}

\begin{abstract}
Multipoint channel charting is a machine learning framework in which multiple massive MIMO (mMIMO) basestations (BSs) collaboratively learn a multi-cell radio map that characterizes the network environment and the users' spatial distribution pattern. The method utilizes large amounts of highdimensional channel state information (CSI) that is passively collected from spatiotemporal samples by the multiple distributed BSs. At each BS, a high-resolution multi-path channel parameter estimation algorithm extracts features hidden in the acquired CSI. Each BS then constructs a local dissimilarity matrix based on the extracted features for its collected samples, and feeds it to a centralized entity which performs feature fusion and manifold learning to construct a multi-cell channel chart. The objective is to chart the radio geometry of a cellular system in such a way that the spatial distance between two users is correlated with their CSI feature distance. The proposed multipoint charting method is capable of unravelling the topology of a Manhattangrid system, and the neighbor relations between CSI features from different spatial locations are captured almost perfectly
\end{abstract}

\section{INTRODUCTION}

Massive multiple-input multiple-output (mMIMO) provides cellular systems with high spectral efficiency [1]. The idea of mMIMO is to equip the infrastructure base-stations (BSs) with hundreds of antennas, which simultaneously serve tens of user equipments (UEs) in the same frequency band. In addition to increasing the spectral efficiency, the high spatial resolution provided by the large-scale antenna arrays used at mMIMO BSs can also be exploited for a range of sensing applications, such as UE positioning and tracking [2]-[4], UE orientation estimation [5], and environment mapping [6], [7].

Channel State Information (CSI) at mMIMO BSs, especially the slowly changing channel mean and covariance matrices, depend continuously on the spatiotemporal locations of UEs, and the effective radio reflectors and scatters in the physical channel. As a result, the aggregate spatiotemporal CSI contains useful information about the network states including the spatial distribution and trajectories of the UEs, neighborhood relationships among the UEs, and handover relationships among neighboring cells. The massive amount of mMIMO CSI, if collected and stored at the BSs, can potentially be leveraged to learn such network state information, which in turn can be exploited to automate cellular radio resource management (RRM) functions.

To leverage mMIMO CSI for RRM functions, reference [2] introduced the concept of channel charting, which analyzes CSI acquired at a single BS with tools from dimensionality reduction and manifold learning in order to construct a channel chart of the wireless channel that relates physical UE location to CSI. The core idea behind channel charting is that UEs which are close in the geographic space would experience similar and correlated CSI (e.g., channel mean and covariance) at a BS.

Channel charting could be performed by each BS independently with the method discussed in [2]. Different BSs would produce differing channel charts for the same UE samples due to different channel realizations and signal-to-noise ratios (SNRs) experienced at different BSs. To support advanced multicell RRM functions, such as handover and multi-connectivity, a single-point chart, however, is insufficient. Furthermore, single-point channel charting may be inaccurate for CSI from cell-edge UEs as their low SNRs may lead to distortions in the charting results. As a remedy to these limitations, we consider multipoint channel charting in which multiple mMIMO BSs collaboratively learn a channel chart for UEs in the multi-cell network. Based on CSI from multiple BSs, multipoint channel charting builds a common multi-cell chart which merges the views of all BSs by applying the concepts of multi-view ML and data fusion. As we will show, multipoint channel charting is able to produce trustworthy channel charts, as it can utilize all CSI information available at multiple BSs and exploit redundancy in multipoint CSI to combat the distortion which occurs in single-point channel charting results.

\section{Multipoint Channel Charting Framework}

A UE moves in two related geometries. The conventional spatial geometry, i.e., the spatial position in the real world. The radio geometry is related to the radio environment between the transmitter and receiver, i.e., represented by CSI of the radio link between the UE and BS. Changes of CSI reflects the location of the UE, and its temporal change in the spatial geometry. Consider a single-antenna UE $k$, transmitting a specific pilot signal $\mathbf{s}_{k, t}$ with a time stamp $t$. A mMIMO BS equipped with $M$ antenna elements receives the pilot the signal for channel estimation. For simplicity, we consider a uniform linear array (ULA) at the BS. Then, for a single coherence bandwidth, the channel vector can be modeled [8] as

$$
\mathbf{h}=\sum_{l=1}^{L} \beta_{l} \mathbf{a}_{\mathrm{BS}}\left(\phi_{l}\right)
$$

where $L$ is the number of multi-path components (including the LoS ray if the channel is in LoS) which are created by the radio scatters, $\phi_{l}$ is the impinging direction-of-arrival (DoA) of the lth propagation path related to the orientation of the BS array, and $\beta_{l}$ a random complex gain for the lth path which depends on the transmit power, path delays, reflection coefficients of the contributing scatters, and impacts of radio chain components. In addition, the vector $\mathbf{a}_{\mathrm{BS}}(\phi)$ represents 


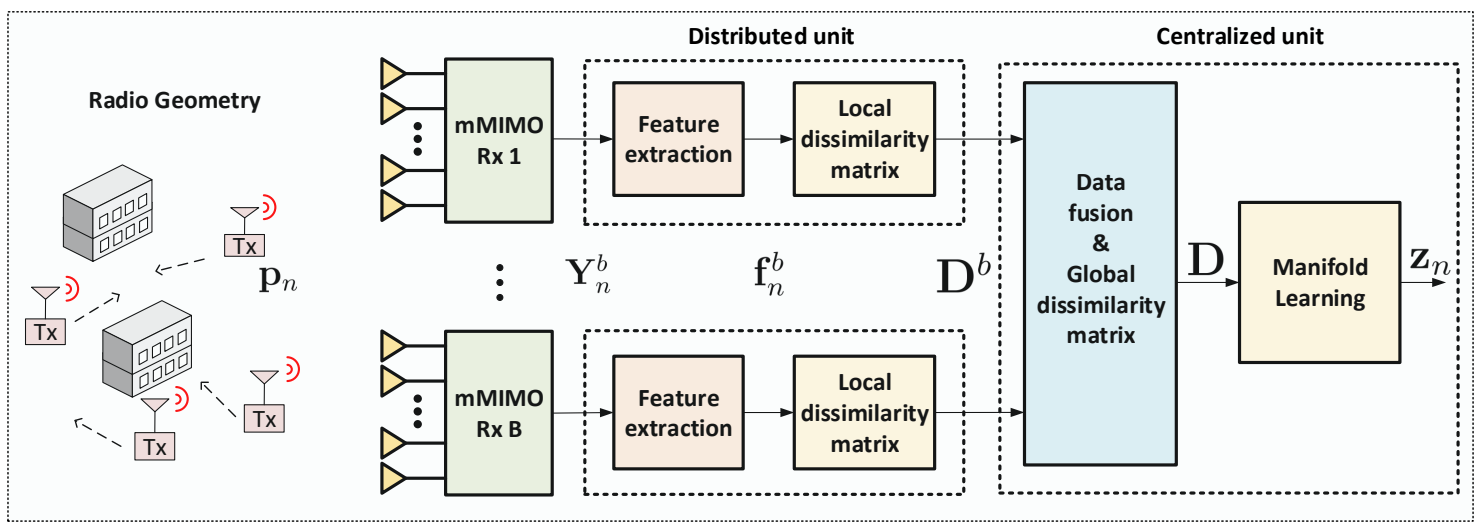

Fig. 1. Multi-point channel charting framework. UE transmitters (Tx) at spatial location $\left\{\mathbf{p}_{n}\right\}_{n=1}^{N}$ send pilot signals to massive MIMO BS receivers (Rx) over the wireless channels. First, channel state information (CSI) $\left\{\mathbf{Y}_{n}^{b}\right\}_{n=1}^{N}$ are estimated by each BS $b \in\{1, \ldots, B\}$, then useful channel features $\left\{\mathbf{f}_{n}^{b}\right\}_{n=1}^{N}$ are extracted from $\left\{\mathbf{Y}_{n}^{b}\right\}_{n=1}^{N}$ at each BS. Each BS then constructs a local view $\mathbf{D}^{b}$ on the dissimilarities for its observed samples. Then, $\mathbf{D}^{b}$ and $\left\{\mathbf{f}_{n}^{b}\right\}_{n=1}^{N}$ are reported to a logical centralized unit $(\mathrm{CU})$ that performs data fusion and manifold learning in order to learn the global channel chart in an unsupervised manner.

the BS array steering vector (ASV) which depends on the geometry of the entire array and the incident angle $\phi$. For a ULA with the plane-wave approximation, the ASV is

$$
\mathbf{a}_{\mathrm{BS}}(\phi)=\left[1, e^{j \frac{2 \pi}{\lambda} s \sin (\phi)}, \ldots, e^{j(M-1) \frac{2 \pi}{\lambda} s \sin (\phi)}\right]^{\mathrm{T}},
$$

where $M$ is the number of $\mathrm{BS}$ antennas, $\lambda$ is the carrier wavelength, and $s$ is the antenna spacing.

Noticing that the DoAs $\left\{\phi_{l}\right\}_{l=1}^{L}$ and the powers of $\left\{\beta_{l}\right\}_{l=1}^{L}$ change much more slowly and smoothly with the UE movements as compared to the coefficients of $\mathbf{h}$, it is worth considering the raw 2 nd moment (covariance) of $\mathbf{h}$ [2], which depends on $\left\{\phi_{l}\right\}_{l=1}^{L}$ and $\left\{\left|\beta_{l}\right|^{2}\right\}_{l=1}^{L}$ as

$$
\begin{aligned}
\mathbf{Y} & =\mathbb{E}\left[\mathbf{h h}^{\mathrm{H}}\right]=\mathbb{E}\left[\sum_{l=1}^{L}\left|\beta_{l}\right|^{2} \mathbf{a}_{\mathrm{BS}}\left(\phi_{l}\right) \mathbf{a}_{\mathrm{BS}}^{\mathrm{H}}\left(\phi_{l}\right)\right] \\
& =\mathbf{A S A}^{\mathrm{H}} \in \mathbb{C}^{M \times M},
\end{aligned}
$$

with $\mathbf{A}=\left[\mathbf{a}_{\mathrm{BS}}\left(\phi_{1}\right) \cdots \mathbf{a}_{\mathrm{BS}}\left(\phi_{L}\right)\right]$ a matrix of the array steering vectors, and $\mathbf{S}=\operatorname{diag}\left(\mathbb{E}\left[\left|\beta_{1}\right|^{2}\right], \ldots, \mathbb{E}\left[\left|\beta_{\mathrm{L}}\right|^{2}\right]\right)$ containing the powers of all multi-path components. In practice, the covariance matrix can be estimated from time/frequency domain samples as $\hat{\mathbf{Y}}=\frac{1}{T} \sum_{t=1}^{T} \hat{\mathbf{h}}_{t} \hat{\mathbf{h}}_{t}^{\mathrm{H}}$, where $T$ is the number of channel realizations one averages over. In wideband channels, the covariance can be calculated across multiple coherence bandwidths.

The spatiotemporal samples $\left\{\mathbf{Y}_{n}^{b}\right\}_{n=1}^{N}$ are the CSI collected at the $b$ th BS $(b=1, \ldots, B)$ from $N$ UE locations $\left\{\mathbf{p}_{n}\right\}_{n=1}^{N}$. In a static radio environment with omnidirectional antennas and fixed transmit power, the covariance depends solely on the UE spatial location. As detailed in [2], a key assumption enabling channel charting is that there is a (statistical) mapping from spatial location $\mathbf{p}_{n}$ to covariance CSI $\mathbf{Y}_{n}^{b}$ measured at the $b$ th $\mathrm{mMIMO}$ BS:

$$
\mathcal{H}^{b}: \mathbb{R}^{d} \rightarrow \mathbb{C}^{D} ; \mathcal{H}^{b}\left(\mathbf{p}_{n}\right)=\mathbf{Y}_{n}^{b},
$$

where $D=M \times M$ is the dimensionality of the radio geometry over $\mathbb{C}$. Thus, for a specific spatial location $\mathbf{p}_{n}$, the related CSI $\mathbf{Y}_{n}^{b}$ is unique. In addition, we assume that this mapping is continuous, i.e., the CSI related to two nearby spatial locations is similar. This mapping depends on the distributions and dielectric properties of the static reflectors, scatterers, and blockages in the radio network environment. Given the CSI samples $\left\{\left\{\mathbf{Y}_{n}^{b}\right\}_{n=1}^{N}\right\}_{b=1}^{B}$ collected at $B$ mMIMO BSs from $N$ unknown random spatial locations $\left\{\mathbf{p}_{n}\right\}_{n=1}^{N}$, we are interested in finding a low-dimensional channel chart $\left\{\mathbf{z}_{n}\right\}_{n=1}^{N}$ with $\mathbf{z}_{n} \in \mathbb{R}^{d}$, such that

$$
\left\|\mathbf{z}_{n}-\mathbf{z}_{m}\right\|_{2} \approx \alpha\left\|\mathbf{p}_{n}-\mathbf{p}_{m}\right\|_{2}, \text { for } n, m \in\{1,2, \ldots, N\},
$$

where $\alpha$ is a common scaling factor.

Figure 1 depicts the overall framework for multi-point channel charting. The $b$ th mMIMO BS collects CSI samples from its coverage area and has its own local view on the distribution of the samples it observes. A feature extraction function that distills useful information $\left\{\mathbf{f}_{n}^{b}\right\}_{n=1}^{N}$ from the raw UE CSI $\left\{\mathbf{Y}_{n}^{b}\right\}_{n=1}^{N}$ is applied. These features $\left\{\mathbf{f}_{n}^{b}\right\}_{n=1}^{N}$ are then used to compute a local dissimilarity matrix $\mathbf{D}^{b}$. The dissimilarity matrix $\mathbf{D}^{b}$ represents the local view on the CSI at BS $b$, related to radio distances between the sampled points. Note that different BSs will have different views on the samples. First, different BSs observe different parts of the entire CSI sample set in the network due to different cellular coverage areas. Second, different BSs may have different opinions on the dissimilarity for the same sample pair due to different channel conditions, e.g., different SNR conditions. One BS may have more reliable information on the CSI of a specific UE than another BSs if the channel conditions between this BS and the UE are better. To construct a global and reliable multi-cell channel chart, we have to fuse the data produced by different BSs. In this regard, a global dissimilarity matrix $\mathbf{D}$ is then constructed based on $\left\{\mathbf{D}^{b}\right\}_{b=1}^{B}$ from the $B$ BSs by a logical centralized unit (CU) that takes into account a reliability factor. Finally, dissimilarity-matrix-based manifold learning is used to generate the multi-cell channel chart. 


\section{CSI FEATURES AND DISSIMILARITY}

In order to learn a channel chart that reflects the spatial locations of the CSI samples, we need a feature that is smoothly and slowly changing with the UE location. In particular, the DoAs $\left\{\phi_{l}\right\}_{l=1}^{L}$ and powers $\left\{\left|\beta_{l}\right|^{2}\right\}_{l=1}^{L}$ of the multi-path components in (1) are such features.

In channel charting, we are interested in the dissimilarities among large-scale CSI samples rather than the physical position of a single UE. Differing from conventional positioning techniques, multi-path effects can be exploited in channel charting as locations close in spatial geometry would have similar multi-path components. By using an elaborate dissimilarity metric for these multi-path features, spatial relationships among the sample locations can be revealed by channel charting algorithms.

\section{A. Feature Extraction via MUSIC}

The MUSIC algorithm [9] is widely used for DoA and frequency spectrum estimation. Here, we consider MUSIC algorithm for channel feature extraction. The input of the algorithm is the covariance matrix of the data and it can be used for arbitrary antenna array geometries, as long as the relative antenna positions are known. Assuming that the number of antennas $M$ is larger than the number of multi-path components $L$, the array steering vectors for the $L$ paths are linearly independent, and that the signal $\mathbf{h}$ and noise $\mathbf{e}$ are orthogonal, the estimated covariance matrix is

$$
\hat{\mathbf{Y}}=\mathbb{E}\left[(\mathbf{h}+\mathbf{e})(\mathbf{h}+\mathbf{e})^{\mathrm{H}}\right]=\mathbf{Y}+\sigma_{e}^{2} \mathbf{I}_{M},
$$

where $\sigma_{e}^{2}$ is the power of each entry of $\mathbf{e}$. The signal covariance matrix $\mathbf{Y}=\mathbf{A S A}^{\mathrm{H}}$ has a rank $L$; it therefore has $L$ eigenvectors with nonzero eigenvalues in the signal subspace, and $M-L$ eigenvectors corresponding to the zero eigenvalue. Let $\mathbf{Y}=\mathbf{U} \boldsymbol{\Lambda} \mathbf{U}^{\mathrm{H}}$ be the eigendecomposition of $\mathbf{Y}$, it can be proved that the estimated covariance matrix has the eigendecomposition as $\hat{\mathbf{Y}}=\mathbf{U}\left(\boldsymbol{\Lambda}+\sigma_{e}^{2} \mathbf{I}_{M}\right) \mathbf{U}^{\mathrm{H}}$ [9]. Based on this eigendecomposition, we can partition the eigenvector matrix $\mathbf{U}$ into two parts as $\mathbf{U}=\left[\mathbf{U}_{\mathrm{s}} \mathbf{U}_{\mathrm{n}}\right]$, where $\mathbf{U}_{\mathrm{s}}$ of size $M \times L$ defines the signal subspace, while $\mathbf{U}_{\mathrm{n}}$ of size $M \times(M-L)$ defines the noise subspace.

The core idea of MUSIC is to find the signal directions using the so-called pseudo-spectrum

$$
p(\phi)=\frac{1}{\mathbf{a}_{\mathrm{BS}}^{\mathrm{H}}(\phi) \mathbf{U}_{\mathrm{n}} \mathbf{U}_{\mathrm{n}}^{\mathrm{H}} \mathbf{a}_{\mathrm{BS}}(\phi)}=\frac{1}{\left\|\mathbf{U}_{\mathrm{n}}^{\mathrm{H}} \mathbf{a}_{\mathrm{BS}}(\phi)\right\|_{2}^{2}} .
$$

If $\phi$ equals one of the DoAs of the multi-path components, the denominator is small. Therefore, the estimated DoAs are the $L$ largest peaks in the pseudo-spectrum. In practice, the number of paths $L$ is unknown, and it is estimated based on the eigendecomposition of $\hat{\mathbf{Y}}$. The path powers are estimated as the $L$ largest eigenvalues in the eigen-decomposition. To pair the estimated DoAs and the path powers, one can check the correlations between the eigenvectors for the powers and the array steering vectors for the DoAs. Finally, we will get a channel feature vector $\mathbf{f}=\left[\lambda_{1}, \lambda_{2}, \ldots, \lambda_{\hat{L}}, \phi_{l_{1}}, \phi_{l_{2}}, \ldots, \phi_{l_{\hat{L}}}\right] \in \mathbb{R}^{2 \hat{L}}$

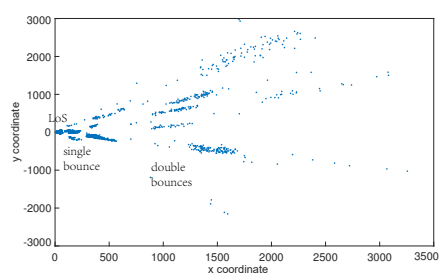

(a) Cartesian points.

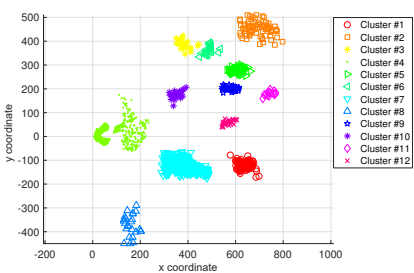

(b) Cartesian points after scaling.
Fig. 2. Cartesian points for multi-path components generated from CSI features extracted via MUSIC by a mMIMO BS for 1000 sampled UE locations in an urban scenario; the origin represents the BS position.

for each BS-to-UE pair, where $\hat{L}$ is the estimated number of significant channel paths.

\section{B. Dissimilarity Metric}

Each BS extracts a feature set $\left\{\mathbf{f}_{n}^{b}\right\}_{n=1}^{N}$ via MUSIC for the sampled UE locations. In what follows, we define a novel dissimilarity metric for the pairs of CSI samples based on the proposed feature. A good dissimilarity metric $d_{f}\left(\mathbf{f}_{n}^{b}, \mathbf{f}_{m}^{b}\right)$ for two CSI samples $n$ and $m$ should be proportional to the real spatial distance $\left\|\mathbf{p}_{n}-\mathbf{p}_{m}\right\|_{2}$.

For two CSI features $\mathbf{f}_{n}^{b}=\left[\lambda_{1}, \ldots, \lambda_{L_{1}}, \phi_{1}, \ldots, \phi_{L_{1}}\right]$, $\mathbf{f}_{m}^{b}=\left[\mu_{1}, \ldots, \mu_{L_{2}}, \theta_{1}, \ldots, \theta_{L_{2}}\right]$, we need to identify the paths for the sample pair that are similar, being created by, for example, the same scatters. For this, we transform the path DoA and power pairs to points in a cartesian coordinate system according to

$$
\mathcal{F}\left(\mathbf{f}_{n}^{b}\right)=\left[\mathbf{x}_{1}, \ldots, \mathbf{x}_{L_{1}}\right], \mathcal{F}\left(\mathbf{f}_{m}^{b}\right)=\left[\mathbf{y}_{1}, \ldots, \mathbf{y}_{L_{2}}\right],
$$

with $\mathbf{x}_{i}=\left[\frac{\cos \phi_{i}}{\sqrt{\lambda_{i}}}, \frac{\sin \phi_{i}}{\sqrt{\lambda_{i}}}\right]^{\mathrm{T}}, \mathbf{y}_{j}=\left[\frac{\cos \theta_{j}}{\sqrt{\mu_{j}}}, \frac{\sin \theta_{j}}{\sqrt{\mu_{j}}}\right]^{\mathrm{T}}$. If the channel rays were known, one could classify these cartesian points into LoS, single-bounce and double-bounce points according to the reflection orders for their corresponding multi-path components. To produce dissimilarity metrics in an unsupervised situation, it is worth noting that nearby UE locations would produce similar cartesian points as they share common scatters and have similar multi-path components.

Figure 2(a) shows the cartesian points generated by a mMIMO BS from 1000 UE locations in an urban scenario. The multi-path components for all sampled UE locations have clustered structures. If two sampled UE locations are close, then they have multi-path components in the same clusters. The clusters of LoS, single-bounce and double-bounce points have different densities; the cluster of LoS points is the densest, while a cluster of double-bounce points is sparser than single-bounce and LoS clusters. To construct a dissimilarity measure, we transform the features $\left\{\mathbf{f}_{n}^{b}\right\}_{n=1}^{N}$ into cartesian points to guarantee that the cartesian points lie in a confined area, as shown in Figure 2(b). Then, a density-based clustering algorithm, such as the density-based spatial clustering of applications with noise (DBSCAN) [10] is adopted to label these cartesian points. Figure 2(b) shows the clustering result for the above 1000 sampled UE locations.

Assuming that the multi-path components for all $N$ sampled UE locations are clustered into $C$ clusters and a component 
$\mathbf{x}$ has a label $l(\mathbf{x}) \in\{1, \ldots, C\}$, the dissimilarity metric for two samples $n$ and $m$ as in (8) is defined as

$d_{f}\left(\mathbf{f}_{n}^{b}, \mathbf{f}_{m}^{b}\right)= \begin{cases}\left\|\mathbf{x}_{i}-\mathbf{y}_{j}\right\|_{2}, & \text { if } n, m \text { share the same clusters, } \\ \left\|\mathbf{x}_{1}-\mathbf{y}_{1}\right\|_{2}, & \text { otherwise, }\end{cases}$

where $[i, j]=\arg \max _{i, j}\left\{\min \left(\lambda_{i}, \mu_{j}\right)\right\}$ satisfying $l\left(\mathbf{x}_{i}\right)=$ $l\left(\mathbf{y}_{j}\right)$. The core idea behind (9) is that if two samples share paths in a same cluster, one should estimate their distance based on these similar paths.

\section{Global Dissimilarity Matrix via Data Fusion}

Finally, to realize multipoint channel charting, we have to fuse the CSI dissimilarities from the multiple BSs related to the dissimilarity of sample UE pairs. Based on (9), each mMIMO BS constructs a dissimilarity matrix $\mathbf{D}^{b}(b \in\{1,2, \ldots, B\})$ with $D_{n, m}^{b}=d_{f}\left(\mathbf{f}_{n}^{b}, \mathbf{f}_{m}^{b}\right)$. These should be merged into one global multi-cell dissimilarity matrix $\mathbf{D}$. Note that signals from a sampled location $\mathbf{p}_{n}$ may not be received by a BS $b$. In this case, we set $\mathbf{f}_{n}^{b}=\varnothing, D_{n, m}^{b}$ and $D_{m, n}^{b}$ for all $m \neq n$ to be a large value $d_{\max }$, and let the $\operatorname{SNR} \gamma_{n}^{b}$ be zero. We shall use the following network-level weighted dissimilarity

$$
D_{n, m}=\left(\sum_{b=1}^{B} w_{b}\right)^{-1} \sum_{b=1}^{B} w_{b} D_{n, m}^{b}
$$

where $w_{b}=\left[\min \left(\gamma_{n}^{b}, \gamma_{m}^{b}\right)\right]^{2}$ is the weight characterizing the reliability of the dissimilarity generated by BS $b$ for samples $n$ and $m$, and a SNR $\gamma_{n}^{b}$ is estimated using $\mathbb{E}\left\{\left\|\mathbf{h}_{n}^{b}\right\|_{2}^{2} / \sigma^{2}\right\}$ with $\sigma^{2}$ as the noise power, and $\mathbf{h}_{n}^{b}$ the channel vector between the BS $b$ and UE location $\mathbf{p}_{n}$.

\section{Channel Charting Algorithms And PERFormance EVALUATION}

Based on the proposed channel features and dissimilarity measure, we then apply different manifold learning algorithms to produce a multi-cell channel chart for the CSI samples. We consider three representative algorithms, i.e., Sammon's Mapping (SM) [11], Laplacian eigenmaps (LE) [12] and tdistributed stochastic neighbor embedding ( $t$-SNE) [13].

We consider an urban outdoor multi-cell mmWave network scenario as depicted in Figure 3. The BSs are below rooftop, and signals will be reflected or blocked by the walls. A raytracing channel model is considered to generate the multipath channels [14]. Table I summarizes the used simulation parameters. We generate $N=5000$ UE locations on the streets of a Manhattan grid, as depicted in Figure 3, and gather estimated CSI samples from them. For SM and $t$-SNE, gradient descent is applied to compute the channel charts, with initializations $\mathbf{Z}_{0}$ drawn from the standard normal distribution. The number of nearest neighbors used in LE and the perplexity for $t$-SNE are chosen to be $N / 20$.

Figure 4 shows the multi-point charting results using SM, LE, and $t$-SNE based on the merged dissimilarities from multiple BSs. Performance of these three algorithms with different sets of participating BSs are investigated. The first row, Figures 4(a), 4(b), and 4(c) shows the results of SM,

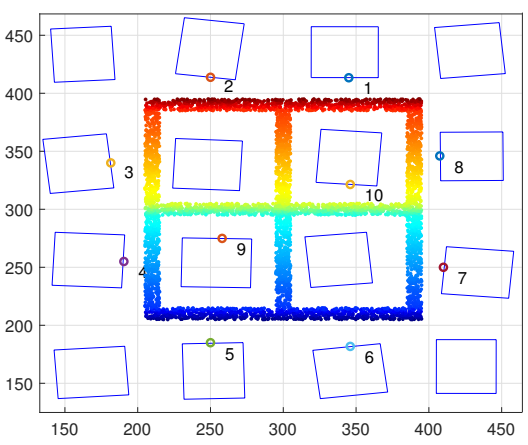

Fig. 3. Simulation scenario in a Manhattan grid with 10 BSs labeled by numbers and sampled UE locations marked by colors.

TABLE I

SIMULATION PARAMETERS OF THE CONSIDERED SYSTEM SCENARIO.

\begin{tabular}{lcc}
\hline Parameter & Symbol & Value \\
\hline Carrier frequency & $f_{c}$ & $28 \mathrm{GHz}$ \\
System bandwidth & $b_{w}$ & $256 \mathrm{MHz}$ \\
OFDM subcarrier number & $N_{c}$ & 256 \\
UE Tx power & $N_{c} \times \rho_{\mathrm{UE}}$ & $23 \mathrm{dBm}$ \\
BS noise power & $N_{c} \times \sigma^{2}$ & $-86 \mathrm{dBm}$ \\
\hline
\end{tabular}

LE and $t$-SNE based on merged dissimilarities from four BSs $\{1,3,5,7\}$. The second row shows results with six BSs $\{1,3,5,7,9,10\}$, while the third row shows results with all 10 BSs. The channel charting performance improves when increasing the number of BSs, for all three algorithms, as more useful information is provided in the merged dissimilarity matrix. All three algorithms provide well-preserved local embeddings. With $t$-SNE, the global structure is well captured even with four participated BSs, as shown in Figure 4(c). It is remarkable that multipoint channel charting is able to perfectly capture the topology of the Manhattan simulation scenario, with ribbon-like streets and holes.

\section{CONClusion}

We have proposed multipoint channel charting, where CSI from multiple massive MIMO BSs is fused to provide a chart of the radio geometry of a multi-cell network. Multiple BSs in the network measure the CSI from individual users, and construct CSI features from these measurements. The features are fused in a central unit, and manifold learning techniques are applied to identify how the 2D (or 3D) spatial geometry of the user locations is embedded in the high-dimensional CSI space. We have developed improved CSI features based on direct estimation of incoming multi-path components, and created a novel dissimilarity measure based on clustering the information related to the estimated DoA and power of all separable multi-path components of set of sample users. We have observed that multi-point channel charting, acting only on received CSI from sample users, can recover the streettopology of the network perfectly

\section{REFERENCES}

[1] T. L. Marzetta, "Noncooperative cellular wireless with unlimited numbers of base station antennas," IEEE Trans. Wireless Commun., vol. 9, no. 11 , pp. 3590-3600, Nov. 2010. 


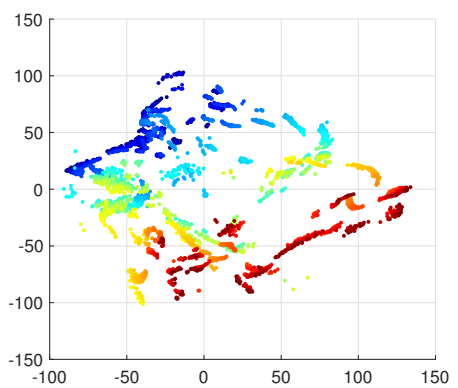

(a) $\mathrm{SM}, 4 \mathrm{BSs}$

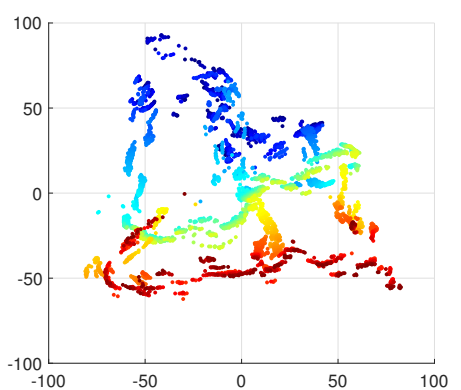

(d) SM, 6 BSs

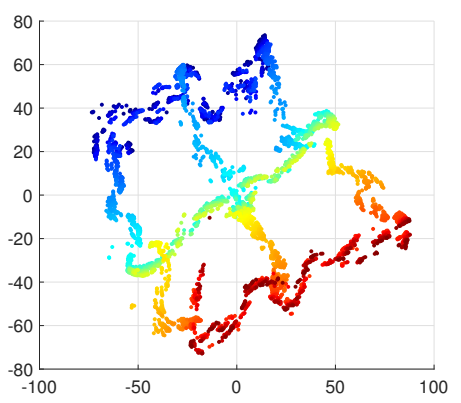

(g) SM, $10 \mathrm{BSs}$

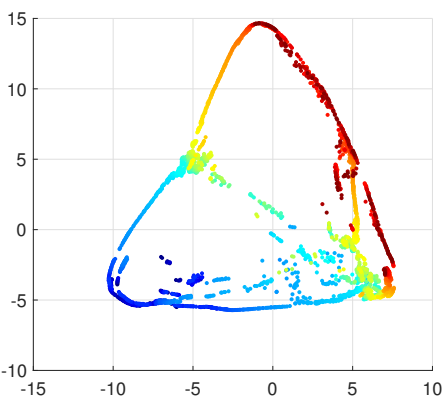

(b) LE, 4 BSs

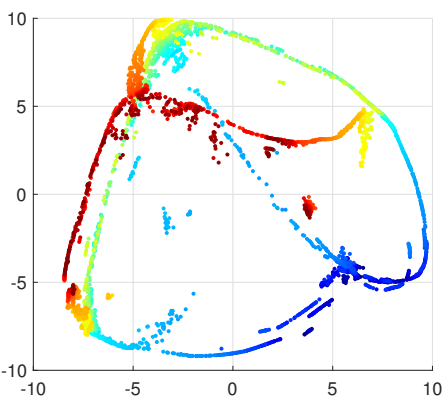

(e) LE, 6 BSs

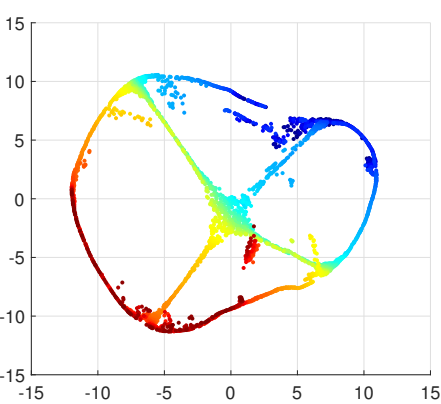

(h) LE, $10 \mathrm{BSs}$

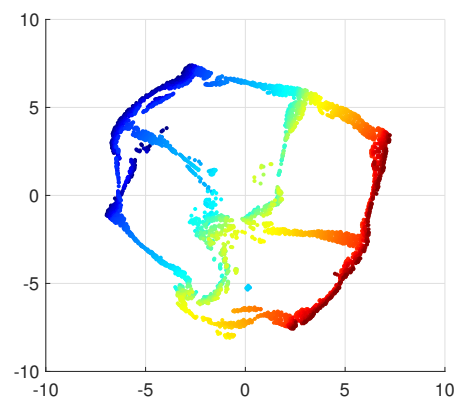

(c) $t$-SNE, 4 BSs

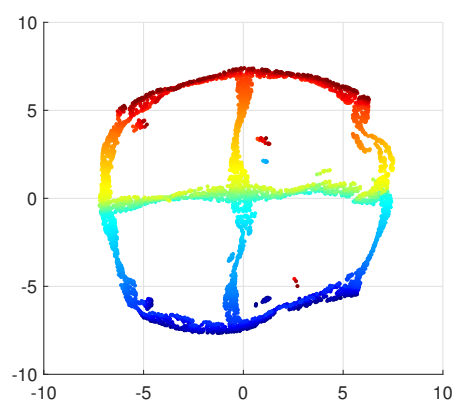

(f) $t$-SNE, $6 \mathrm{BSs}$

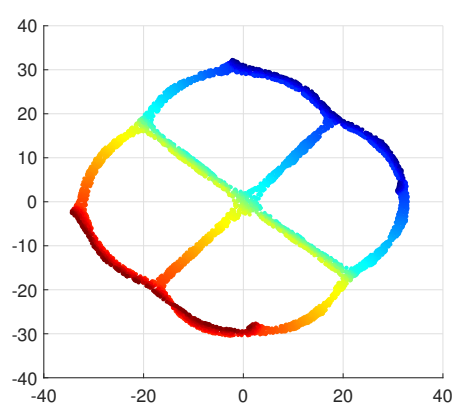

(i) $t$-SNE, $10 \mathrm{BSs}$

Fig. 4. Multipoint charting results via SM, LE and $t$-SNE based on channel features from a) BSs 1, 3, 5, 7; b) BSs 1, 3, 5, 7, 9, 10; and c) all 10 BSs. The multipoint charting performance improves with more participating BSs.

[2] C. Studer, S. Medjkouh, E. Gönültas, T. Goldstein, and O. Tirkkonen, "Channel charting: Locating users within the radio environment using channel state information," IEEE Access, vol. 6, pp. 47 682-47698, 2018.

[3] N. Garcia, H. Wymeersch, E. G. Larsson, A. M. Haimovich, and M. Coulon, "Direct localization for massive MIMO," IEEE Trans. Sig. Proc., vol. 65, no. 10, pp. 2475-2487, May 2017.

[4] A. Guerra, F. Guidi, and D. Dardari, "Single-anchor localization and orientation performance limits using massive arrays: MIMO vs. beamforming," IEEE Trans. Wireless Commun., June 2018.

[5] A. Shahmansoori, G. E. Garcia, G. Destino, G. Seco-Granados, and H. Wymeersch, "Position and orientation estimation through millimeterwave MIMO in 5G systems," IEEE Trans. Wireless Commun., vol. 17, no. 3, pp. 1822-1835, Mar. 2018.

[6] F. Guidi, A. Guerra, and D. Dardari, "Millimeter-wave massive arrays for indoor SLAM," in IEEE Int. Conf. on Commun. (ICC), June 2014, pp. 114-120.

[7] F. Guidi, A. Guerra, D. Dardari, A. Clemente, and R. D'Errico, "Environment mapping with millimeter-wave massive arrays: System design and performance," in IEEE Globecom Workshops (GC Wkshps), Dec. 2016, pp. 1-6.

[8] M. R. Akdeniz, Y. Liu, M. K. Samimi, S. Sun, S. Rangan, T. S. Rappaport, and E. Erkip, "Millimeter wave channel modeling and cellular capacity evaluation," IEEE Journal on Selected Areas in Communications, vol. 32, no. 6, pp. 1164-1179, June 2014.
[9] R. Schmidt, "Multiple emitter location and signal parameter estimation," IEEE Transactions on Antennas and Propagation, vol. 34, no. 3, pp. 276-280, Mar 1986.

[10] M. Ester, H.-P. Kriegel, J. Sander, and X. Xu, "A density-based algorithm for discovering clusters a density-based algorithm for discovering clusters in large spatial databases with noise," in Proceedings of the Second International Conference on Knowledge Discovery and Data Mining, ser. KDD’96. AAAI Press, 1996, pp. 226-231.

[11] J. W. Sammon, "A nonlinear mapping for data structure analysis," IEEE Transactions on Computers, vol. C-18, no. 5, pp. 401-409, May 1969.

[12] M. Belkin and P. Niyogi, "Laplacian eigenmaps and spectral techniques for embedding and clustering," in Advances in neural information processing systems, 2002, pp. 585-591.

[13] L. v. d. Maaten and G. Hinton, "Visualizing data using t-SNE," Journal of machine learning research, vol. 9, no. Nov, pp. 2579-2605, 2008.

[14] S. Hur, S. Baek, B. Kim, J. Park, A. F. Molisch, K. Haneda, and M. Peter, " $28 \mathrm{GHz}$ channel modeling using 3D ray-tracing in urban environments," in 9th European Conference on Antennas and Propagation (EuCAP), May 2015, pp. 1-5. 